\title{
Der Ersatz des Chlors durch Brom und Jod bei der Trennung des Cers von den anderen Ceriterden. Von
}

\author{
Philip E. Browning und Edwin I. Roberts. ${ }^{1}$
}

Eines der am besten bekannten Verfahren zur 'Trennung des Cers von Lanthan und Didym ist das von Mosander, ${ }^{2}$ welches darin besteht, dals man in eine Suspension der Erdhydroxyde in Ätzalkalihydroxydlösung Chlor einleitet, bis die Lösung gesättigt ist und die Flüssigkeit nicht mehr auf Lackmus alkalisch reagiert. Unter diesen Bedingungen bleibt fast alles Cer als Ceribydroxyd ungelöst, während die anderen Ceriterden leicht in Lösung gehen. Bei der Behandlung von gemischtem Material hält der Rückstand von Ceribydroxyd meist einige Ceriterden zurück, so dafs die Behandlung mit Cblor wiederholt werden mufs. Dies Verfahren bietet zwei Nachteile, nämlich die Bereitung und Anwendung von Chlorgas und die Lösungswirkung der bei der Reaktion gebildeten Chlorwasserstoffsäure auf das Ceribydroxyd: $2 \mathrm{Ce}(\mathrm{OH})_{3}+\mathrm{Cl}_{2}=2 \mathrm{CeO}_{2}+$ $2 \mathrm{HCl}+2 \mathrm{H}_{2} \mathrm{O}$.

Die zu beschreibende Untersuichung wurde unternommen, um den Einflufs des Ersatzes von Chlor durch Brom und Jod festzustellen. Ein vorläufiger Versuch wurde ausgeführt, indem man einen Niederschlag ron ausgewaschenen Hydroxyden der Ceriterden in Wasser suspendierte, etwas Brom zusetzte und die Reaktion unter gelegentlichem Umrühren vor sich gehen liels. Der Niederschlag nahm die Farbe von Cerihydroxyd an und beim Filtrieren fand man, dafs das Filtrat beträchtliche Mengen Ceriterden, jedoch ohne Cer enthielt.

Bei den folgenden Versuchen wurden Lösungen mit einem bekannten Gehalt an gemischten Oxyden, die aus etwa 50\% Cer und $50 \%$ der anderen Ceriterden bestanden, mit einem geringen Überschufs von Natrium- oder Kaliumhydroxyd behandelt. Die Suspension der Berlin.

1 Aus dern Amer. Journ. Sci. (Sill.) ins Deutsche übertragen von I. KoppeL-

IJ prakt. Chom. 30, 267. 
Hydroxyde in der alkalischen Flüssigkeit wurde mit flüssigem Brom oder Bromwasser in deutlichem Überschufs behandelt und das Gemisch auf ein Dampfbad gesetzt, bis der grölsere Teil des freien Broms ausgetrieben war. Der Rückstand wurde dann abfiltriert, gewaschen und wie vorher behandelt. Dies Verfahren wiederholte man zweimal, wobei man fand, dafs das Filtrat die in der Tabelle angegebenen Mengen ron Ceriterden frei von Cer enthielt. Der Rückstand von der letzten Behandlung zeigte nach dem Auflösen in Säuren nur schwach die Didymbanden. Bei Versuch 6 war die Andeutung für die Gegenwart von Didym nur sehr gering. Bei einem weiteren Versuch wurden wie bei 5,6 and $10 \mathrm{~g}$ des Materiales noch einer vierten und fünften Behandlung mit Brom unterworfen, wobei die vierte Behandlung nur einen kleinen Bruchteil eines Grammes an Oxyden gab, während die fünfte nur wenige Milligramme lieferte. In beiden Fällen waren diese Oxyde frei von Cer. Die Oxyde aus den ersten Filtraten waren viel heller in der Farbe als die aus den letzten, woraus hervorgeht, dals das Lanthan durch das Brom leichter aufgelöst wird, als das Didym. Die Ergebnisse folgen in der Tabelle.

Tabelle.

\begin{tabular}{c|c|c|c|c|c}
\hline \hline & $\begin{array}{c}\text { Gemischte Oxyde } \\
\text { angew. } \\
\mathrm{g}\end{array}$ & $\begin{array}{c}\text { Gefundene Oxyde } \\
\text { im 1. Filtrat } \\
\mathrm{g}\end{array}$ & $\begin{array}{c}\text { im 2. Filtrat } \\
\mathrm{g}\end{array}$ & $\begin{array}{c}\text { im 3. Filtrat } \\
\mathrm{g}\end{array}$ & $\begin{array}{c}\text { Gef. Gesamt- } \\
\text { menge der } \\
\text { Oxyde, } \mathrm{g}\end{array}$ \\
\hline \hline 1 & 1.0000 & 0.3310 & 0.0720 & 0.0190 & 0.4420 \\
2 & 1.0000 & 0.2900 & 0.1010 & 0.0420 & 0.4330 \\
3 & 1.0000 & 0.2250 & 0.1290 & 0.0640 & 0.4180 \\
4 & 1.0000 & 0.2750 & 0.0860 & 0.0740 & 0.4350 \\
5 & 10.0000 & 3.1360 & 1.0050 & 0.5930 & 4.7340 \\
6 & 10.0000 & 3.4590 & 0.5240 & 0.8560 & 4.8390
\end{tabular}

Hieraus ergibt sich, dafs durch Ersatz des Chlors durch Brom beim MosaNder schen Verfabren 50\% der anderen Ceriterden in einer Operation rom Cerihydroxyd getrennt werden können, und dafs nach drei Wiederholungen praktisch alle anderen Erden entfernt sind, ohne dals das Cerihydroxyd angegriffen ist.

Die Vorteile der Methode bestehen in der leichten Anwendbarkeit des Broms und in dem Fehlen der Neigung der Bromwasserstoffsäure zum Auflösen von Cerihydroxyd.

Ein Versuch wurde mit Jod an Stelle von Brom ausgefübrt: 
die gefallten und suspendierten Hydroxyde von $2 \mathrm{~g}$ der gemischten Oxyde wurden mit $1 \mathrm{~g}$ festem Jod behandelt. Nach 2 stündigem Stehen auf dem Dampfbad entfernte man das überschïssige Jod durch Kochen und filtrierte das verbleibende Hydroxyd ab. Das Filtrat gab $0.0980 \mathrm{~g}$ cerfreie Oxyde von schwach brauner Furbe. Dies zeigt, dals die Einwirkung ron Jod im allgemeinen die gleiche ist wie die von Chlor und Brom, dafs sie aber zu unvollständig verläuft, um praktisch anwendbar zu sein.

New Haven, U. S. A., The Kent Chemical Laboratory of Yale University.

Bei der Redaktion eingegangen am 11. August 1909.

\section{Druckfehlerberich tigung.}

Im Autorenregister Band 63, Seite 354 ist die Aufführung der Abhandlung von W. Herz und A. BorLs, Gleichgewichtsresktionen mit Wismuthydroxyd 63, 282 vergessen worden.

Ferner ist auf S. 353 hinter Andrews einzufigen: ANdrews und FARR, Die volumetrische Bestimmung kleiner Arsenmengen 62, 123.

Im Sachregister Band 6:, Seite 359 bei ,Wismuthydroxyd, Verhalten gegen Alkalien" lies statt 61, 387 richtig 63, 282. 